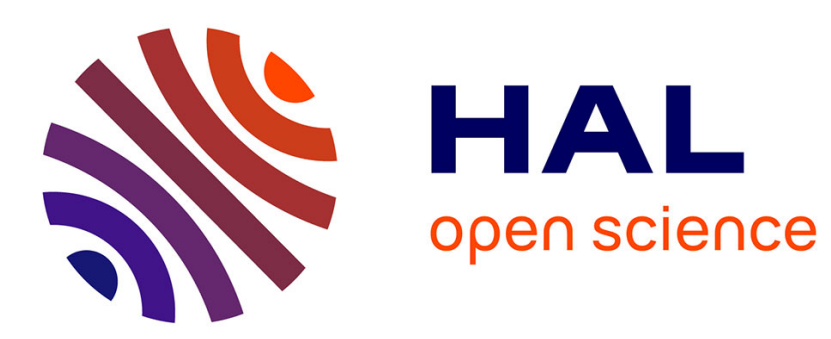

\title{
One-dimensional models of growing and coalescing droplets
}

\author{
I. Yekutieli, C. Godrèche, Bernard Derrida
}

\section{To cite this version:}

I. Yekutieli, C. Godrèche, Bernard Derrida. One-dimensional models of growing and coalescing droplets. Physica A: Statistical Mechanics and its Applications, 1992, 185 (1), pp.240-244. 10.1016/0378-4371(92)90462-Y . hal-03285627

\section{HAL Id: hal-03285627 \\ https://hal.science/hal-03285627}

Submitted on 19 Jul 2021

HAL is a multi-disciplinary open access archive for the deposit and dissemination of scientific research documents, whether they are published or not. The documents may come from teaching and research institutions in France or abroad, or from public or private research centers.
L'archive ouverte pluridisciplinaire HAL, est destinée au dépôt et à la diffusion de documents scientifiques de niveau recherche, publiés ou non, émanant des établissements d'enseignement et de recherche français ou étrangers, des laboratoires publics ou privés. 


\title{
One-dimensional models of growing and coalescing droplets
}

\author{
I. Yekutieli ${ }^{\mathrm{a}}$, C. Godrèche ${ }^{\mathrm{a}}$ and B. Derrida ${ }^{\mathrm{b}}$ \\ "Service de Physique de l'Etat Condensé, Centre d'Etudes de Saclay, \\ 91191 Gif-sur-Yvette Cedex, France \\ 'Service de Physique Théorique, Centre d'Etudes de Saclay, 91191 Gif-sur-Yvette Cedex, \\ France
}

\begin{abstract}
We introduce extremely simple geometric models of a system of growing and coalescing droplets, in order to study its time evolution. For a one-dimensional surface we write down equations for the evolution of the distributions of distances between droplets in these models. We calculate these distributions in the long time limit, where we find a scaling regime.
\end{abstract}

In recent years, considerable studies [1-4] have been performed on the dynamics of condensation of water on a non-wetting surface. Experimentally, water vapor passed over a cold surface, condenses on the surface in the form of droplets. It is observed that each droplet grows with time, due to the continual condensation of water, and that when two droplets come in contact, they coalesce, because of surface tension, into a single droplet. This is a particular case of aggregation where the spatial extent of the clusters (on a $2 \mathrm{D}$ surface) is governed by their (3D) form. The interesting feature of this form of condensation is that after some time from the beginning of an experiment, the system attains a regime of self-similarity in time: the distribution of droplet sizes has a scaling form, and the fraction of the surface covered by the droplets (the "coverage") is constant.

A plausible model for this system consists in placing $D$-dimensional droplets randomly on a $d$-dimensional surface (in reality, $D=3$, and $d=1$ or 2 ). The diameter, $d_{i}(t)$, of each droplet is taken to grow in time as a power law

$$
d_{i}(t)=d_{i}(t)^{\mu}
$$

and when two droplets come in contact, they are replaced instantaneously by one droplet with a diameter given by mass conservation,

$$
d_{i+j}+\left(d_{i}^{D}+d_{j}^{D}\right)^{1 / D}
$$

placed at the center of mass of the coalescing droplets. 
For the sake of simplicity, we considered only the one-dimensional version of this model [5,6], in which case the relevant quantities are droplet diameters, $d_{i}$, distances between the centers of neighboring droplets ("intervals"), $h_{i}$, and gaps between droplets, $g_{i}$. Numerical simulations show that this model reaches a self-similar regime. In fig. 1 we plot distributions of sizes of gaps, diameters and intervals, scaled by the average droplet diameter at different times in the scaling regime, for the model defined above with $D=3$. The coverage remains at a constant value of 0.8 throughout this regime.

These results remain unchanged qualitatively for different values of $D$. An interesting case is found by taking the limit $D \rightarrow \infty$ where the distribution of diameters becomes a $\delta$-function.

Next, we consider a simplified model of growth and coalescence where all droplets have the same diameter and grow at the same rate. When two droplets touch, they are replaced by a single droplet of the same size centered in the middle between the coalescing droplets. This model can be interpreted as purely geometric if we consider the points which are centers of the droplets. We trace only coalescences, and this consists in searching for the two nearest points (separated by the shortest interval, which we denote by $h_{0}$ ) and replacing them by a single point in the middle between them. The droplets

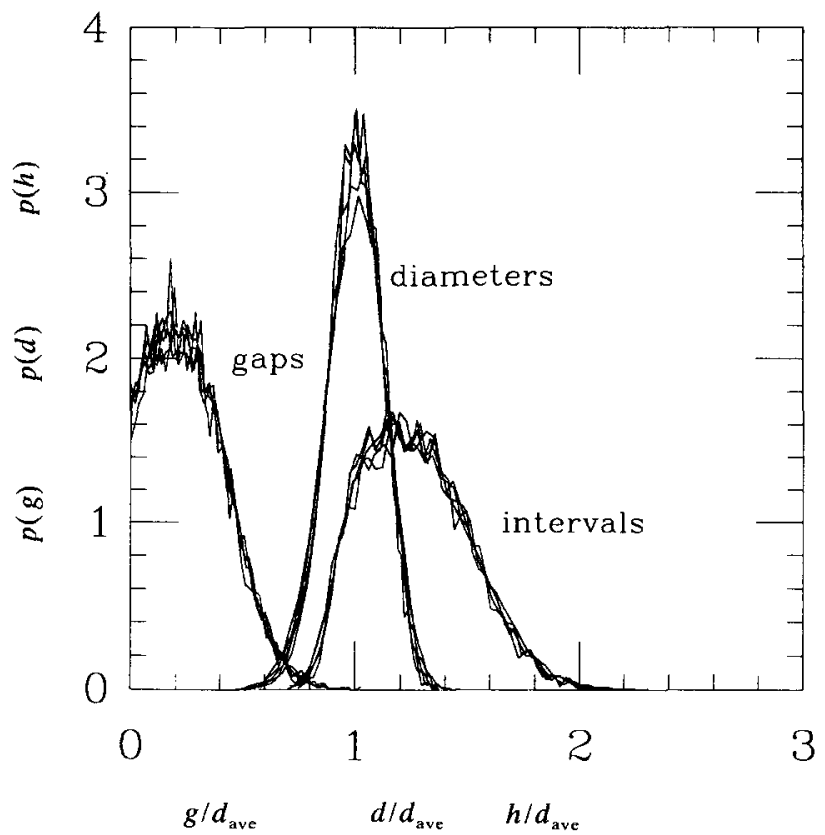

Fig. 1. 3D droplet model simulation: distributions of gaps, diameters and intervals, scaled by the average diameter. 


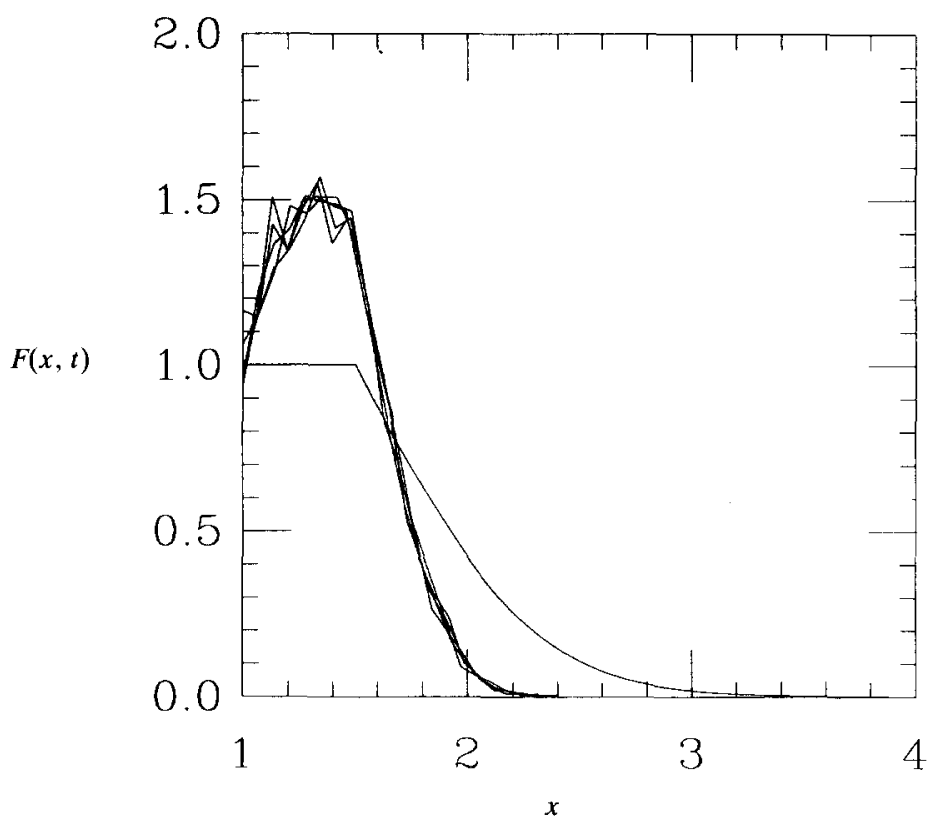

Fig. 2. Geometric model I: scaled distributions of interval lengths (simulation, mean field).

diameters are taken to be all equal to $h_{0}$. This model exhibits a self-similar regime, and in fig. 2 we plot distributions of interval lengths at different times in the scaling regime, scaled by $h_{0}$. The corresponding coverage is 0.72 .

We now study the evolution of a sequence of intervals along the line, where at each step we take the shortest interval $h_{0}$, cut it in two, and add each half to one of its neighbors. As a first step, we take a mean-field approximation where we assume that the intervals are uncorrelated. This can be visualized as having a sack with sticks in it, and that at each step one takes the shortest stick, cuts it in two and add each of the pieces to two other sticks chosen at random from the sack. Defining $f_{t}(h) \delta h$ as the probability of finding an interval of length between $h$ and $h+\delta h$ at time $t$, we can express $f_{t+\delta t}(h)$ after a small time step $\delta t$ during which all intervals of length between $h_{0}$ and $h_{0}+\delta h_{0}$ have been coalesced as

$$
f_{t+\delta t}(h)=f_{t}(h)+\left[-f_{t}(h)+2 f_{t}\left(h-\frac{1}{2} h_{0}\right) \theta\left(h-\frac{3}{2} h_{0}\right)\right] f_{t}\left(h_{0}\right) \delta h_{0} .
$$

This equation gives rise in the long time limit, for most initial conditions, to a particular distribution of interval lengths, scaling in time as $h_{0}$, plotted in fig. 2 , the corresponding coverage being 0.64 .

As $h_{0}$ is a natural length scale in this problem, we introduce a scaled distribution $F(x, t)$ defined by 


$$
f_{t}(h)=h_{0}^{-1} F\left(h / h_{0}, t\right)
$$

which obeys, when taking the limits $\delta t \rightarrow \infty$ and $\delta h_{0} \rightarrow \infty$, a partial differential equation

$$
\begin{aligned}
(a+t) \frac{\partial F(x, t)}{\partial t}= & F(x, t)+x \frac{\partial F(x, t)}{\partial x} \\
& +F(1, t)\left[2 F\left(x-\frac{1}{2}, t\right) \theta\left(x-\frac{3}{2}\right)-F(x, t)\right],
\end{aligned}
$$

where it is taken that $h_{0}(t)=a+t$. We do not have the complete time dependent solution of eq. (5), but we can calculate the family of stationary solutions of this equation, parametrized by a continuous parameter $F(1)$. We can also argue why for most initial conditions, the stationary solution that will be chosen (assuming one is reached) is the one corresponding to $F(1)=1$, which is the solution displayed in fig. 2. As is seen clearly in this figure, this solution is not at all satisfactory. The reason for this is that correlations do exist between the intervals, due to the particular coalescence mode chosen. We can make a better approximation for the description of this model by including nearest neighbor correlations, but correlations extend farther than that.

We introduce a second geometric model, similar to the previous one, except for the coalescence mode: when the shortest interval is found, it is added as a whole to either of its neighbors at random, with equal probability. The distribution of interval lengths found in the scaling regime (here too!) of the evolution of this model, is plotted in fig. 3 , scaled by $h_{0}$, with a coverage of 0.56 .

Using the same notations as for the previous model, we can express the time evolution of $f_{t}(h)$ by

$$
f_{t+\delta t}(h)=f_{t}(h)+f_{t}\left(h-h_{0}\right) \theta\left(h-2 h_{0}\right) f_{t}\left(h_{0}\right) \delta h_{0},
$$

and the partial differential equation obeyed by $F(x, t)$,

$$
(a+t) \frac{\partial F(x, t)}{\partial t}=F(x, t)+x \frac{\partial F(x, t)}{\partial x}+F(1, t) F(x-1, t) \theta(x-2)
$$

It should be noted that for this model there are no correlations between neighboring intervals, and the mean field is exact! Again we find that for most initial conditions, eq. (6) leads to a scaling solution which corresponds to the stationary solution of eq. (7) with $F(1)=1$, also plotted in fig. 3, overlapping the simulation results.

Apart from the generic stationary solution with $F(1)=1, F(1)$ can take any 


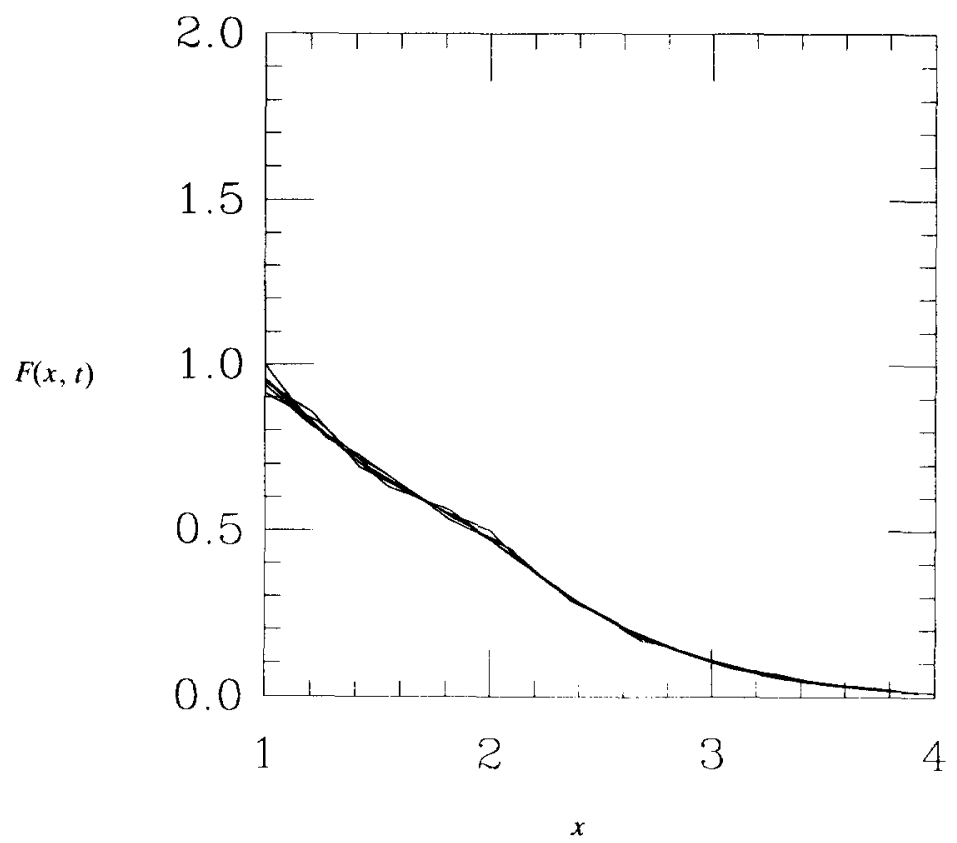

Fig. 3. Geometric model II: scaled distributions of interval lengths (simulation, mean field).

value between 0 and 1 . These solutions, which decay as a power law for $x \gg 1$, correspond to slowly decaying initial distributions, with the same power law behavior. This distinction is analogous to the distinction made in the addition of independent random variables between the Gaussian law and Lévy laws [7].

\section{References}

[1] D. Beysens and C.M. Knobler, Phys. Rev. Lett. 57 (1986) 1433.

[2] J.L. Viovy, D. Beysens and C.M. Knobler, Phys. Rev. A 37 (1988) 4965.

[3] F. Family and P. Meakin, Phys. Rev. A 40 (1989) 3836.

[4] A. Steyer, P. Guénoun, D. Beysens, D. Fritter and C.M. Knobler, Europhys. Lett. 12 (1990) 211.

[5] B. Derrida, C. Godrèche and I. Yekutieli, Europhys Lett. 12 (1990) 385.

[6] B. Derrida, C. Godrèche and I. Yekutieli, Phys. Rev. A, to appear.

[7] P. Lévy, Théorie de l'addition des variables aléatoires (Gauthier-Villars, Paris, 1954). 